\title{
Detectability of deuterated water in prestellar cores ${ }^{\star}$
}

\author{
D. Quénard ${ }^{1,2}$, V. Taquet ${ }^{3}$, C. Vastel ${ }^{1,2}$, P. Caselli ${ }^{4}$, and C. Ceccarelli ${ }^{5,6}$ \\ 1 Université de Toulouse, UPS-OMP, IRAP, 31028 Toulouse Cedex 4, France \\ e-mail: dquenard@irap.omp.eu \\ 2 CNRS, IRAP, 9 Av. colonel Roche, BP 44346, 31028 Toulouse Cedex 4, France \\ 3 Leiden Observatory, Leiden University, PO Box 9513, 2300 RA Leiden, The Netherlands \\ ${ }^{4}$ Max-Planck Institute for Extraterrestrial Physics, Giessenbachstrasse 1, 85748 Garching, Germany \\ ${ }^{5}$ Université Grenoble Alpes, IPAG, 38000 Grenoble, France \\ ${ }^{6}$ CNRS, IPAG, 38000 Grenoble, France
}

Received 20 July 2015 / Accepted 23 October 2015

ABSTRACT

\begin{abstract}
Context. Water is an important molecule in the chemical and thermal balance of dense molecular gas, but knowing its history throughout the various stages of the star formation is a fundamental problem. Its molecular deuteration provides us with a crucial clue to its formation history. $\mathrm{H}_{2} \mathrm{O}$ has recently been detected for the first time towards the prestellar core L1544 with the Herschel Space Observatory with a high spectral resolution (HIFI instrument).

Aims. Prestellar cores provide the original reservoir of material from which future planetary systems are built, but few observational constraints exist on the formation of water and none on its deuteration before the collapse starts and a protostar forms at the centre. We report on new APEX observations of the ground state $1_{0,1}-0_{0,0} \mathrm{HDO}$ transition at $464 \mathrm{GHz}$ towards the prestellar core L1544. The line is undetected, and we present an extensive study of the conditions for its detectability in cold and dense cloud cores.

Methods. The water and deuterated water abundances have been estimated using an advanced chemical model simplified for the limited number of reactions or processes that are active in cold regions $(<15 \mathrm{~K})$. In this model, water is removed from the gas phase by freezing onto dust grains and by photodissociation. We use the LIME radiative transfer code to compute the expected intensity and profile of both $\mathrm{H}_{2} \mathrm{O}$ and $\mathrm{HDO}$ lines and compare them with the observations.

Results. The predicted $\mathrm{H}_{2} \mathrm{O}$ line intensity of the LIME model using an abundance and structure profile, coupled with their dust opacity, is over-estimated by a factor of $\sim 3.5$ compared to the observations. We present several ad hoc profiles that best-fit the observations and compare the profiles with results from an astrochemical modelling, coupling gas phase and grain surface chemistry. The water deuteration weakly depends on the external visual extinction, the external ISRF, and contraction timescale. The $[\mathrm{HDO}] /\left[\mathrm{H}_{2} \mathrm{O}\right]$ and $\left[\mathrm{D}_{2} \mathrm{O}\right] /\left[\mathrm{H}_{2} \mathrm{O}\right]$ abundance ratios tend to increase towards the centre of the core up to $25 \%$ and $\sim 8 \%$, respectively.

Conclusions. Our comparison between observations, radiative transfer, and chemical modelling shows the limits of detectability for singly deuterated water, through the ground-state transitions $1_{0,1}-0_{0,0}$ and $1_{1,1}-0_{0,0}$ at 464.9 and $893.6 \mathrm{GHz}$, respectively, with both single-dish telescope and interferometric observations. This study also highlights the need of a detailed benchmark amongst different radiative transfer codes for this particular problem of water in prestellar cores.
\end{abstract}

Key words. ISM: abundances - astrochemistry - line: identification - ISM: molecules - ISM: individual objects: L1544

\section{Introduction}

Water is an important molecule not only on Earth but also in space. Indeed, since it is formed by the two most abundant elements in the Universe that bind in molecules, water governs the chemical composition and the thermal balance of the interstellar dense molecular gas. This is also the gas from which stars are formed, so that water influences the whole star formation process at various levels in the different phases (e.g. Caselli $\&$ Ceccarelli 2012). In molecular clouds, iced water is present in large quantities, up to half the oxygen elemental abundance, and is synthesised on the interstellar grains (e.g. Boogert et al. 2015). In the denser regions inside the molecular clouds, which are the prestellar cores that will eventually form stars, water is still mostly iced (Caselli et al. 2012). The same water, formed in those first two stages, is then found in the gas phase where the dust is warm enough $(\geq 100 \mathrm{~K})$ in the hot cores, hot corinos, and

* Molecular line data (FITS cube) are only available at the CDS via anonymous ftp to cdsarc.u-strasbg. fr (130.79.128.5) or via http://cdsarc.u-strasbg.fr/viz-bin/qcat?J/A+A/585/A36 protostellar molecular shocks (e.g. van Dishoeck et al. 2014). It is then again iced and gaseous in the different warm and cold, respectively, zones of the protoplanetary disks, where planets form (e.g. Carr \& Najita 2008; Podio et al. 2013). Finally, water is the major component of the volatiles in comets (e.g. Bockelée-Morvan et al. 2014).

In summary, we see water throughout all the stages of the solar-type star forming process up to the leftovers of this process, represented by comets and meteorites. Nevertheless, we do not fully know its whole history: how it evolves from molecular clouds to comet ices and, perhaps, to terrestrial oceans. A crucial aspect in reconstructing the water history is provided by its deuteration by water molecules with one or two deuterium atoms. This is because molecular deuteration is very sensitive to the moment the molecule is formed, the temperature, and also the environment. And since water changes continuously from ice to vapour, it keeps memory of most, if not all, its past formation history (e.g. Ceccarelli et al. 2014). So far, the water deuteration has been measured in only a handful of objects, all of them warm: hot cores, hot corinos, and protostellar molecular 
shocks (e.g. Coutens et al. 2012, 2013, 2014; Taquet et al. 2013b; Persson et al. 2014). In cold molecular clouds, only the upper limits of the iced deuterated water exist (Dartois et al. 2003; Parise et al. 2003; Aikawa et al. 2012). In prestellar cores, no attempt to measure the deuterated water has been published so far.

This article presents the first upper limit on $\mathrm{HDO} / \mathrm{H}_{2} \mathrm{O}$ in a prestellar core, L1544 (Sect. 2), by combining Herschel observations (Caselli et al. 2012) with new observations obtained at the APEX telescope (Sect. 3). We also report a radiative transfer and chemical study of the ortho- $\mathrm{H}_{2} \mathrm{O}$ and $\mathrm{HDO}$ fundamental lines in L1544 (Sects. 4 and 5, respectively), and show that the two HDO fundamental lines at 464 and $893 \mathrm{GHz}$ lines are not observable with the present facilities. A final section (Sect. 6) summarises the results.

\section{The prestellar core L1544}

L1544 is a prototypical starless core in the Taurus molecular cloud complex $(d \sim 140 \mathrm{pc})$ on the verge of gravitational collapse (Caselli et al. 2002a and references within). It is characterised by high density in its centre (peak density of $2 \times$ $10^{7} \mathrm{~cm}^{-3}$; Keto \& Caselli 2010), low temperature ( $7 \mathrm{~K}$; Crapsi et al. 2007), and high CO depletion in its centre, accompanied by a high degree of molecular deuteration (Caselli et al. 2003; Crapsi et al. 2005; Vastel et al. 2006).

Its physical and dynamical structure has recently been reconstructed by Caselli et al. (2012) and Keto et al. (2014) using the numerous existing observations towards L1544. Among them, the recent detection of water vapour by the Herschel Space Observatory is spectacular because it represents the very first water detection in a prestellar core (Caselli et al. 2010, 2012). The first of these two Herschel observations was made with the wide-band spectrometer (WBS) with a spectral resolution of 1.1 MHz, and water vapour was detected in absorption against the weak dust continuum radiation $(\sim 10 \mathrm{mK})$ in the cloud. Follow-up observations using the High Resolution Spectrometer (HRS) confirmed the absorption and even detected an inverse P-Cygni emission line profile, too narrow to be seen by the WBS, which was predicted by theoretical modelling (Caselli et al. 2010).

This detection provided crucial information for reconstructing the physical and chemical structure of L1544. Indeed, this inverse P-Cygni profile, which is characteristic of gravitational contraction, confirmed that L1544 is on the verge of collapsing. Based on the line shape, Caselli et al. (2012) predict that water is largely frozen into the grain mantles in the interior $(\leq 4000 \mathrm{au})$ of the $\mathrm{L} 1544$ core, where the gaseous $\mathrm{H}_{2} \mathrm{O}$ abundance (with respect to $\mathrm{H}_{2}$ ) is $<10^{-9}$. This level of water vapour is believed to be caused by non-thermal desorption processes such as (a) the photo-desorption of water molecules from the icy mantles by the far UV photons created locally by the interaction of cosmic rays with $\mathrm{H}_{2}$ molecules and the far UV starlight; and (b) the exothermicity of the grain surface chemical reactions that releases the products (e.g. $\mathrm{H}_{2} \mathrm{O}$ and $\mathrm{HDO}$ ) in the gas phase (Vasyunin \& Herbst 2013; Wakelam et al. 2014). Farther away from the centre $\left(\sim 10^{4} \mathrm{AU}\right)$, where the density is low enough $\left(\leq 10^{4} \mathrm{~cm}^{-3}\right)$ for the photo-desorption rate not to be overcome by the freezeout rate, the gaseous $\mathrm{H}_{2} \mathrm{O}$ abundance reaches $\sim 1 \times 10^{-7}$, in agreement with predictions from comprehensive chemical models (Hollenbach et al. 2009).

\section{Observations}

We observed the HDO fundamental transition $1_{0,1}-0_{0,0}$ on April 16, 17, 28, 29, and 30, 2013 and November 2 and 3, 2013 towards L1544 $\left(\alpha_{2000}=05^{\mathrm{h}} 04^{\mathrm{m}} 17.21^{\mathrm{s}}, \delta_{2000}=25^{\circ} 10^{\prime} 42.8^{\prime \prime}\right)$ using the heterodyne instrument (APEX-3) of the APEX observatory. The frequency was centered at $464.92452 \mathrm{GHz}$ to reach the $1_{0,1}-0_{0,0}$ ground-state HDO transition, and the RPG eXtended bandwidth Fast Fourier Transform Spectrometer (XFFTS) backend was used to obtain the highest frequency resolution needed for a comparison with the $\mathrm{H}_{2} \mathrm{O}$ profile. To be consistent with the $\mathrm{H}_{2} \mathrm{O} \mathrm{Herschel/HIFI}$ observations, we mapped the HDO transition within the Herschel/HIFI 40" beam and reached an rms of $50 \mathrm{mK}$ in about $0.1 \mathrm{~km} \mathrm{~s}^{-1}$ velocity bin, averaging all positions in order to compare both observations. Weather conditions were excellent between 0.2 and $0.7 \mathrm{~mm}$ of precipitable water vapour with system temperatures less than $1000 \mathrm{~K}$. Line intensities are expressed in units of main-beam brightness temperature with a main beam efficiency of $60 \%{ }^{1}$. The ortho- $\mathrm{H}_{2} \mathrm{O}\left(1_{10}-1_{01}\right)$ line observation was taken from Caselli et al. (2012) and was observed with Herschel/HIFI. The dust continuum emission flux at $557 \mathrm{GHz}$ is $10.2 \pm 0.2 \mathrm{mK}$ and the rms noise level is $3.8 \mathrm{mK}$ in the spectrum.

\section{4. $\mathrm{H}_{2} \mathrm{O}$ modelling}

\subsection{LIME radiative transfer model}

We ran several radiative transfer modellings of the water emission using LIME, a 3D radiative transfer code (Brinch \& Hogerheijde 2010) based on ALI (accelerated lambda iteration) calculations. Created from RATRAN-1D (Hogerheijde \& van der Tak 2000), LIME does a full radiative transfer treatment in two steps. The first one is to compute the population level of every molecular transition found in the input collision file of the desired molecule. The collisional excitation rates for ortho- $\mathrm{H}_{2} \mathrm{O}$ are not the same if we consider a collision with ortho- $\mathrm{H}_{2}$ or para- $\mathrm{H}_{2}$. In the case of L1544, we assume that all the hydrogen is in the para state as required by recent chemical models to produce the high deuterium fraction observed in cold, dense clouds (Flower et al. 2006; Pagani et al. 2007; Troscompt et al. 2009; Sipilä et al. 2013; Kong et al. 2015). We used the o- $\mathrm{H}_{2} \mathrm{O}-\mathrm{p}-\mathrm{H}_{2}$ collision value from Dubernet et al. (2009), and we assumed a $\mathrm{H}_{2} \mathrm{O}$ ortho-to-para ratio of 3 .

To calculate the population level, LIME needs the 3D structure of the source defined in each point of a model as a function of its Cartesian coordinates $(X, Y, Z)$. The source model is usually defined by a few thousand points distributed randomly among a desired radius. Each point is the centre of a 3D cell, and LIME defines the physical properties of the model in each 3D cells (density, temperature, velocity profile, etc.). At least 10000 points are required to construct the model in order to prevent undersampling. Below 10000 points, neighbouring 3D cells will be created with different sizes, and nonhomogeneous effects will appear in the resulting image after the ray-tracing. Thanks to its random distribution, each one of the 10000 points is located at a unique radius, thus uniquely defined by its physical property. The distribution of points across the model as a function of the radius follows a power law type distribution. This leads to an increasing number of model points per unit volume towards the centre of the cloud, since a finer sampling is needed where the volume densities are higher.

In the second steps, LIME performs a ray-tracing to output the desired image, depending on the user choice of spatial and spectral resolution and of the number of channels, for instance. As a result, a hyper-spectral cube is created for each chosen line

http://www . apex-telescope.org/telescope/efficiency/ 


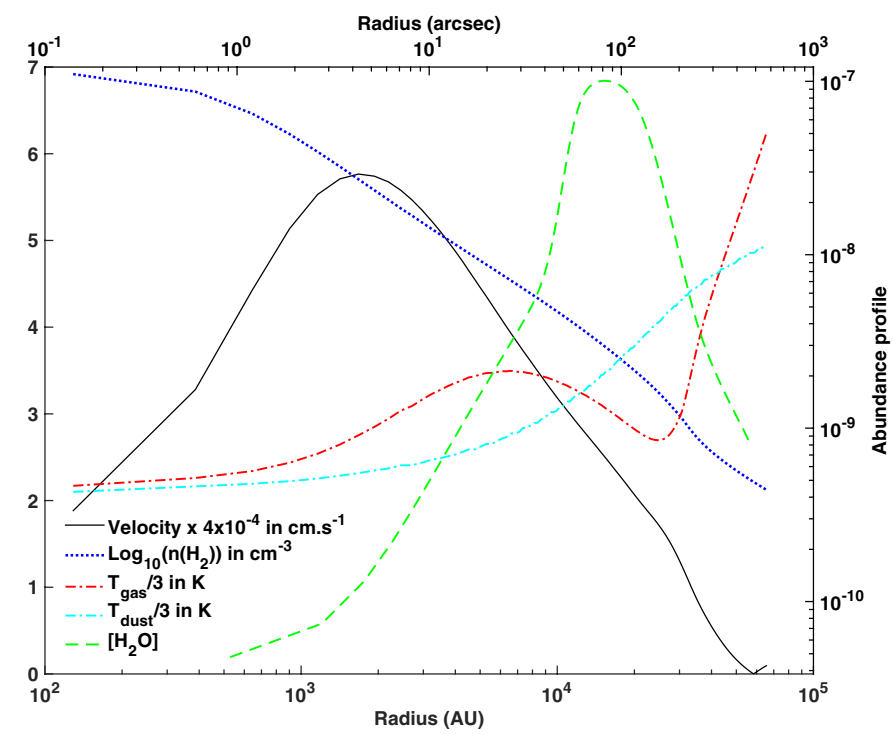

Fig. 1. Gas and dust temperature, density, velocity, and water abundance profiles from Keto et al. (2014). The abundance profile is plotted on a logarthmic scale on the right axis of the figure.

transition. To finish, this cube can be convolved with the beam of the telescope to obtain the spectrum.

The large Einstein coefficient $\left(3.45 \times 10^{-3} \mathrm{~s}^{-1}\right)$ of the $\mathrm{H}_{2} \mathrm{O} 1_{1,0}-1_{0,1}$ transition results in optical depths across the core up to a thousand, depending on excitation, leading to a very nonlinear relationship between the opacity and the column density. This opacity effect slows down the computation of the population level of the line and can lead to a wrong excitation. Keto et al. (2014) used MOLLIE (Keto 1990; Keto \& Rybicki 2010) to fit their Herschel/HIFI data. For the particular problem of water in L1544, MOLLIE has been modified to treat the radiative transfer with an escape probability method. This method optimises the calculation for the optically thick, but highly subthermally excited $\mathrm{H}_{2} \mathrm{O}$ line towards L1544. Therefore, LIME and MOLLIE are two distinct ways to treat the water modelling problem, and it is necessary to model both $\mathrm{H}_{2} \mathrm{O}$ and HDO with LIME in order to make a consistent comparison. More information about the differences between MOLLIE and a full radiative transfer code such as LIME will be discussed in a dedicated paper.

\subsection{Grid results}

We used the physical structure as derived by Keto et al. (2014) to solve the molecular excitation of the $\mathrm{H}_{2} \mathrm{O}$ transition. Figure 1 shows the structure for a slowly contracting cloud in quasi equilibrium. To reproduce the observed line broadening, we considered a Doppler parameter $\beta$ ranging between 100 and $300 \mathrm{~m} \mathrm{~s}^{-1}$. We found that a value of $200 \mathrm{~m} \mathrm{~s}^{-1}$ gives the best line width fit compared to the observations. This result is consistent with the low gas temperature of L1544.

Because of the absorption feature in the $\mathrm{H}_{2} \mathrm{O}$ observation, it is important to take the continuum value derived from the observations into account and compare its value to the modelling. The LIME code can deal with different input dust opacity files as a function of wavelength such as the tabulated files from Ossenkopf \& Henning (1994). The observed continuum value at $557 \mathrm{GHz}$ (or $538.2 \mu \mathrm{m}$ ) is $10.2 \pm 0.2 \mathrm{mK}$ (see Caselli et al. 2012), and the best model value found with LIME is $10.0 \mathrm{mK}$ with $\kappa_{557}=8.41 \mathrm{~cm}^{2} \mathrm{~g}^{-1}$. Keto \& Caselli (2010) based their
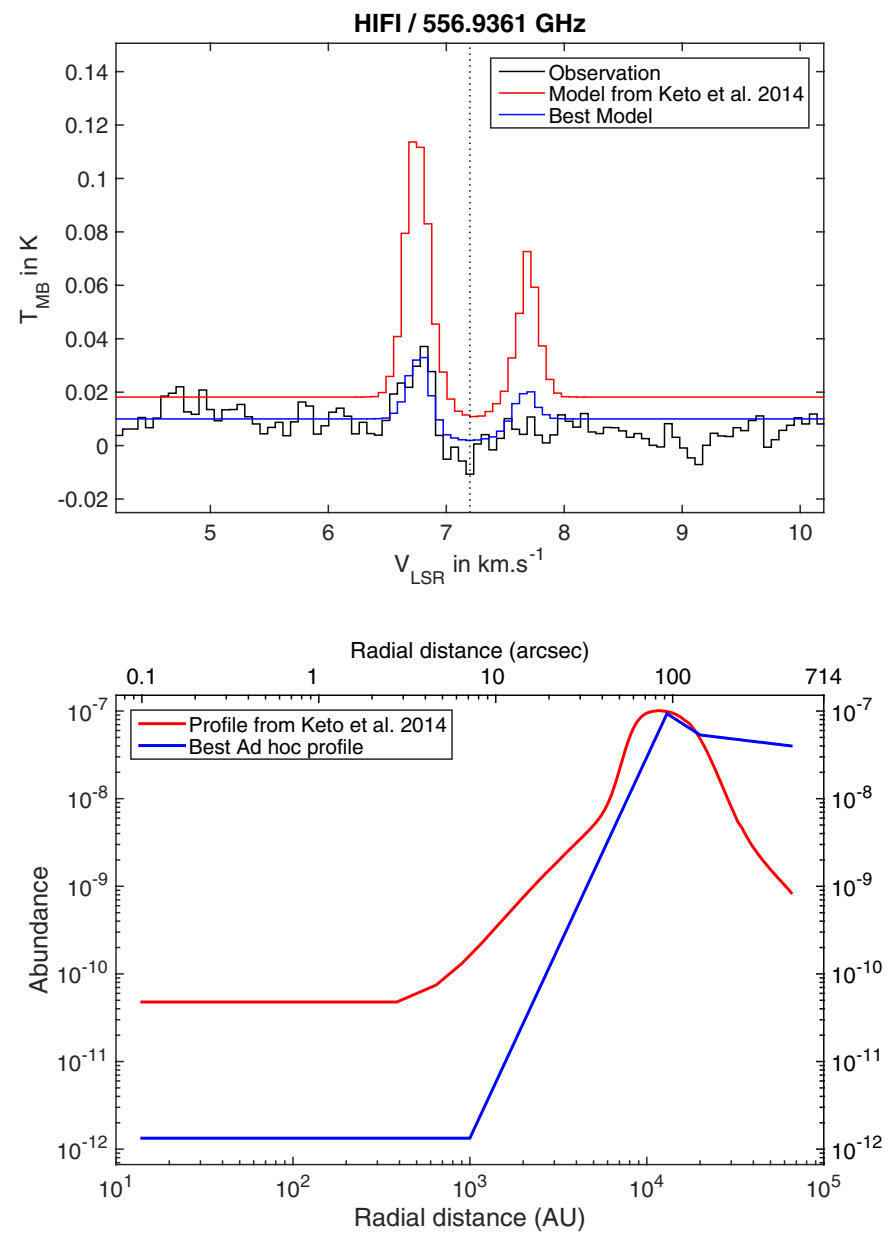

Fig. 2. Top panel: comparison between observations and modelling obtained by LIME using the structure and abundance profile from Keto et al. (2014; in red). The best fit model is shown in blue, using the same structure but an ad hoc abundance profile. Bottom panel: abundance profile from Keto et al. (2014) in red and best fit ad hoc abundance profile in blue as a function of the radial distance from the core in arcsec (top axis) and AU (bottom axis).

dust opacity value on the results found by Zucconi et al. (2001) where the authors approximate the grain opacities of Ossenkopf \& Henning (1994) by piecewise power laws. Keto \& Caselli (2010) derived a value of $\kappa_{557}=3.83 \mathrm{~cm}^{2} \mathrm{~g}^{-1}$ thanks to the following equation describing one of these piecewise power laws (see Appendix B of Zucconi et al. 2001):

$\kappa_{v}=\frac{Q_{v}}{m\left(\mathrm{H}_{2}\right)} \times\left(\frac{\lambda_{\mathrm{a}}}{\lambda_{\mathrm{H}_{2} \mathrm{O}}}\right)^{\alpha} \times\left(\frac{m_{\mathrm{gas}}}{m_{\mathrm{dust}}}\right)$,

with $Q_{v}=3.3 \times 10^{-26} \mathrm{~cm}^{2} \mathrm{H}_{2}^{-1}$ at $\lambda_{\mathrm{a}}=1060 \mu \mathrm{m}, m\left(\mathrm{H}_{2}\right)=3.35 \times$ $10^{-24} \mathrm{~g}$, and $\alpha=2.0$. They had to increase the dust opacity value by a factor of four $\left(\kappa_{557}=15.3 \mathrm{~cm}^{2} \mathrm{~g}^{-1}\right)$ to be able to reproduce the low temperatures measured by Crapsi et al. (2007) towards the centre of L1544.

The result from the LIME modelling with the abundance and structure profile from Keto et al. (2014), coupled with their dust opacity value, is shown in Fig. 2. The $\mathrm{H}_{2} \mathrm{O}$ emission is over-estimated by a factor of $\sim 3.5$, and the dust continuum emission is overestimated by a factor of $\sim 2$ compared to observations. The latter effect is due to the higher dust opacity $\left(\kappa_{557}=15.3 \mathrm{~cm}^{2} \mathrm{~g}^{-1}\right)$ they used compared to the one we found in our best fit $\left(\kappa_{557}=8.41 \mathrm{~cm}^{2} \mathrm{~g}^{-1}\right)$. As discussed above, the difference in the intensity of the line can be explained by the different 
radiative transfer treatments in MOLLIE and LIME, including different grids describing the same physical structure, which will be investigated in a forthcoming paper.

Although we note that Keto \& Caselli (2010) and Keto et al. (2014) present a self-consistent model, where a simple chemistry is followed during dynamical and thermal evolution, and where the dynamical evolution is constrained by previous observations of $\mathrm{N}_{2} \mathrm{H}^{+}$and $\mathrm{CO}$ isotopologues, we decided here to explore possibly different water abundance profiles to find a good match to the $\mathrm{H}_{2} \mathrm{O}$ observations using LIME. For this purpose, we modified the water abundance profile found by Keto et al. (2014), while using their physical structure constrained with the many data published for many years towards this source. The inverse P-Cygni profile is a combination of blue-shifted emission and red-shifted absorption, split in velocity by the inward gas motion in front and at the rear of the cloud. In L1544, the emission is shifted with respect to the absorption by less than a line width, leading to an asymmetry in the line profile. Therefore, only a complex modelling can tentatively disentangle between the blue-shifted emission and red-shifted absorption. The emission is produced in the inner region of the cloud, where the density approaches the critical density of the transition. Meanwhile the absorption layer is located in the outer part of the cloud, where the water abundance peaks.

Several ad hoc profiles have been tested through grid calculations to find the best fit shown in Fig. 2, varying the following different parameters located in four distinct regions of the core:

1. The first region is the inner abundance and we found a best fit value of $\left[\mathrm{H}_{2} \mathrm{O}\right]=1.3 \times 10^{-12}$ at the centre of the core until a radius of $1000 \mathrm{AU}$. Keto et al. (2014) found a value of this inner abundance close to $\left[\mathrm{H}_{2} \mathrm{O}\right]=5 \times 10^{-11}$. If we increase the inner abundance to this value, we also change the slope of the abundance profile, probing a region where the velocity field is higher. This effect leads to a wider and slightly blueshifted emission compared to our best fit. Nonetheless, due to the large HIFI beam at $557 \mathrm{GHz}$, the constraints on the inner abundance are relatively poor. Thus, the contribution of cosmic-ray-induced UV photons responsible for this inner abundance is also poorly constrained, and a proper benchmark between LIME and other radiative transfer codes is needed to develop a deeper analysis of this inner abundance. Cosmic-ray-induced UV photons also appear important for reproducing the observations of $\mathrm{CO}$ isotopologues toward L1544 (Keto \& Caselli 2010).

2. The second region defines the peak abundance value of the external layer. We varied the abundance value and the distance from the centres of the core of this region. We found a best fit value of $\left[\mathrm{H}_{2} \mathrm{O}\right]=9.3 \times 10^{-8}$ at a radius of $13000 \mathrm{AU}$.

3. The third region marks the end of the external layer and, along with the first region, we varied the abundance value and the distance from the centre of the core. We found a best fit value of $\left[\mathrm{H}_{2} \mathrm{O}\right]=5.3 \times 10^{-8}$ at a radius of $20000 \mathrm{AU}$.

4. We finally varied the abundance value at the end of the profile, located at the same final radius of the physical structure of the core given by Keto et al. (2014). We found a best fit value of $\left[\mathrm{H}_{2} \mathrm{O}\right]=4 \times 10^{-8}$. This result may imply that $\mathrm{L} 1544$ is well embedded in a relatively large filament in Taurus, so that the extinction $A_{\mathrm{V}}$ is high enough to at least partially shield interstellar UV photons (see Sect. 4.3).

We decided to first determine the first and second regions at the same time thanks to the grid. We then fixed the third one, which appeared to be independent of the other two. We derived the best fit from these previous models with the help of the standard $\chi^{2}$ minimization value for a spectrum of $N$ points, given by the equation (Coutens et al. 2012):

$$
\chi^{2}=\sum_{j=1}^{N} \frac{\left(I_{\mathrm{obs}, j}-I_{\mathrm{model}, j}\right)^{2}}{\mathrm{rms}^{2}+\left(\mathrm{cal} \times I_{\mathrm{obs}, j}\right)^{2}},
$$

where $I_{\mathrm{obs}}$ and $I_{\text {model }}$ are the observed and the modelling intensity, $\mathrm{cal}_{i}$ is the calibration factor of the spectrum, and $N$ is the total number of points of the spectrum. In the left-hand panels of Fig. 3 we show a small variation in the peak abundance value at the best fit distance of $13000 \mathrm{AU}$ from the centre of the core. It is interesting to note that a little variation in the abundance value at this distance can cause a notable difference in the line profile. Meanwhile, if we change the abundance value at the best fit distance of $20000 \mathrm{AU}$ of the external layer (see right panels of Fig. 3), the emission is the same for the three profiles.

In fact, emission from a molecule like water with high critical densities is only possible in regions with sufficiently high $\mathrm{H}_{2}$ densities. In L1544, this region corresponds to the back part of the core approaching the centre (thus moving towards us) and revealing infall through an inverse P-Cygni profile (emission in the blue-shifted part of the line). We are seeing differences in the height of the blue peak by changing the abundance of water around $13000 \mathrm{AU}$ owing to more or fewer water molecules being present in the outer envelope to be able to absorb the emission coming from the central regions. This results does not contradict the detection of methanol and complex organic molecules (COMs) at radii of about $10000 \mathrm{AU}$ from the centre (Vastel et al. 2014; Bizzocchi et al. 2014), because water, methanol, and COMs have to be present in the gas phase at such radii. However, water at the volume densities present in the outer envelope can only absorb, while methanol and other complex molecules can emit more easily (as their critical densities are not as high). If the water abundance value at the edge of the core is lower than $\left[\mathrm{H}_{2} \mathrm{O}\right] \simeq 1 \times 10^{-8}$, the absorption feature will not be deep enough.

\subsection{Chemical modelling of $\mathrm{H}_{2} \mathrm{O}$ and $\mathrm{HDO}$}

We modelled the abundance profile of standard and deuterated water in L1544 using the GRAINOBLE astrochemical model, described in Taquet et al. (2012, 2014). Briefly, GRAINOBLE couples the gas phase and grain surface chemistry with the rate equation approach introduced by Hasegawa et al. (1992) during the static contraction of a starless core. The gas-phase chemical network was taken from the KIDA database and has been extended to include the spin states of $\mathrm{H}_{2}, \mathrm{H}_{2}^{+}, \mathrm{H}_{3}^{+}$, and the deuterated isotopologues of hydrogenated species with four or fewer atoms among with species involved in the gas phase chemical network of water, ammonia, formaldehyde, and methanol. A more detailed description of the chemical network is presented in Taquet et al. (2014). We also considered the following gasgrain processes:

1. Accretion of gas phase species on the surface of spherical grains with a fixed diameter $a_{\mathrm{d}}$ assumed to be equal to $0.1 \mu \mathrm{m}$.

2. Diffusion of adsorbed species via thermal hopping, exponentially depending on the diffusion-to-binding energy ratio $E_{\mathrm{d}} / E_{\mathrm{b}}$. We set $E_{\mathrm{d}} / E_{\mathrm{b}}=0.65$, following our previous studies.

3. Reaction between two particles via the LangmuirHinshelwood mechanism. 

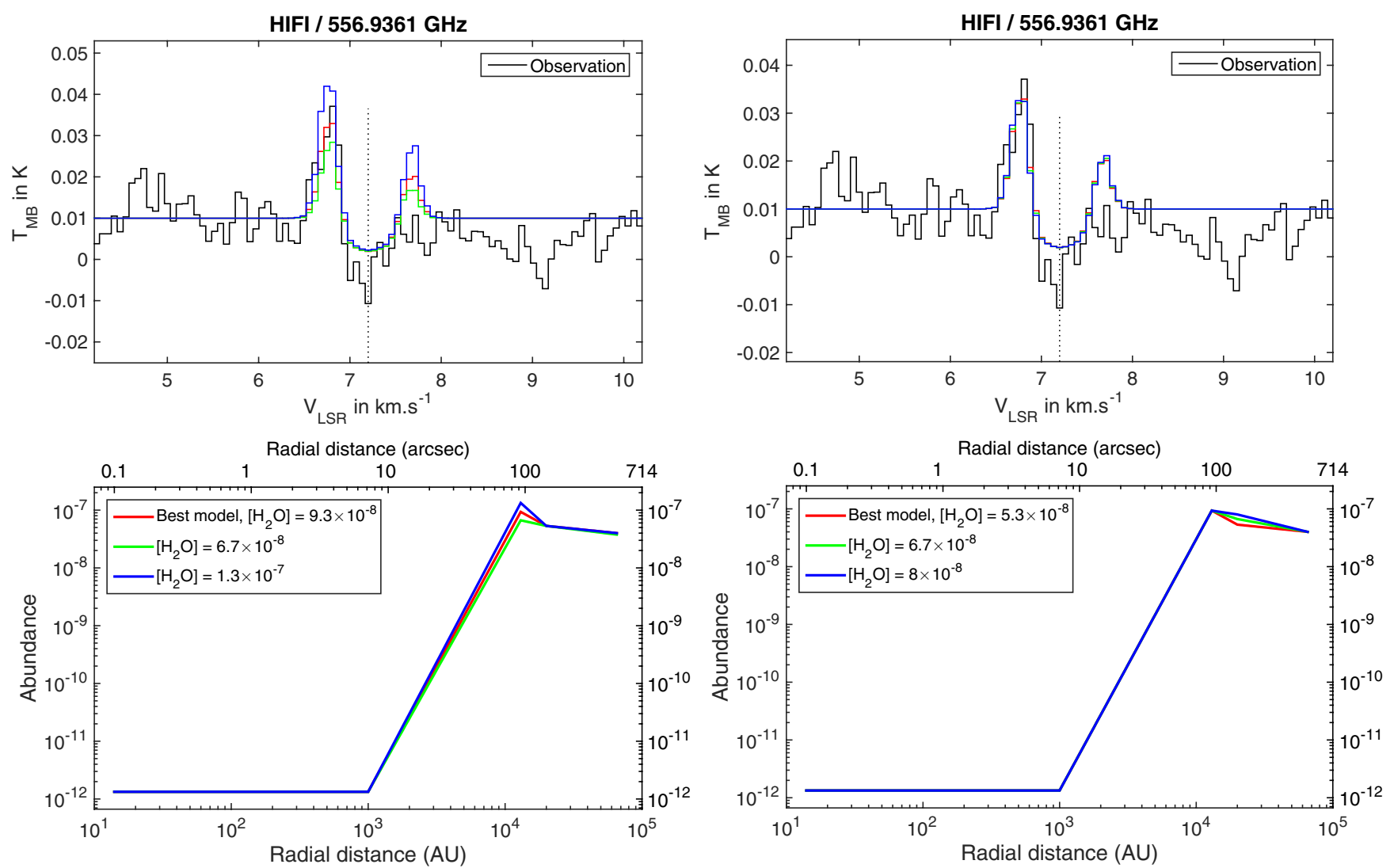

Fig. 3. Top panels: line profile versus observation for three different modellings. The best fit model is shown in red. In the left panel, the abundance value at $13000 \mathrm{AU}$ varies between $6.7 \times 10^{-8}$ and $1.3 \times 10^{-7}$ and for the right panel, the abundance value at $20000 \mathrm{AU}$ varies between $5.3 \times 10^{-8}$ and $8 \times 10^{-8}$. Bottom panel: $\mathrm{H}_{2} \mathrm{O}$ abundance profile used for these respective models as a function of the radial distance from the core in arcsec (top axis) and AU (bottom axis).

4. Desorption of adsorbed species into the gas phase by thermal evaporation, interstellar plus cosmic-ray induced heating of grains, chemical evaporation, and UV photolysis. The thermal evaporation exponentially depends on the binding energy of each species $E_{\mathrm{b}}$ relative to the substrate (see Taquet et al. 2014, for a list of binding energies used in the model). The cosmic-ray-induced heating of grains follows the approach by Hasegawa \& Herbst (1993a) and is adapted to the binding energies considered in this work. The approach adopted for the UV photolysis follows the method described in Taquet et al. (2013a).

We used the multi-layer approach developed by Hasegawa \& Herbst (1993b) to follow the multi-layer formation of interstellar ices and considered three sets of differential equations: one for gas-phase species, one for surface species, and one for bulk species. The equations governing chemical abundances on the surface and in the bulk are linked by an additional term that is proportional to the rate of growth or loss of the grain mantle. As a consequence, surface species are continuously trapped in the bulk because of the accretion of new particles.

We followed the formation and the deuteration of the main ice species following the surface chemical network developed by Taquet et al. (2013a), which is based on laboratory experiments showing the efficient formation of interstellar ice analogues at low temperatures. Transmission probabilities for key reactions involved in the water chemical network have been estimated through quantum chemistry.

The gas-grain chemistry is followed during the static contraction of a dense core starting from a homogeneous translucent sphere of a initial density $n_{\mathrm{H}, \text { ini }}=3 \times 10^{3} \mathrm{~cm}^{-3}$ and a maximal radius of $3 \times 10^{4} \mathrm{AU}$. During the static contraction, the core keeps a Plummer-like density profile:

$$
n_{\mathrm{H}}=\frac{n_{\mathrm{H}, 0}}{\left(1+\left(r / R_{\mathrm{f}}\right)^{2}\right)^{\eta / 2}},
$$

where $n_{\mathrm{H}, 0}$ is the central density, and $R_{\mathrm{f}}$ the characteristic radius inside which the density is uniform. The contraction ends when the density profile reaches the observed profile of L1544, with the following parameters: $n_{\mathrm{H}, 0}=1.8 \times 10^{7} \mathrm{~cm}^{-3}, R_{\mathrm{f}}=450 \mathrm{AU}$, and $\eta=2.1$. Since $R_{\mathrm{f}}$ is given by the product of the sound speed and the free-fall time of the central density, $R_{\mathrm{f}}$ decreases with $1 / \sqrt{n_{\mathrm{H}, 0}}$. Intermediate central densities, and the associated timescale needed to reach them, have been chosen to have a total contraction timescale of about one million years, following observational estimates of molecular cloud cores (e.g. Brünken et al. 2014).

We used the radiative transfer code DUSTY (Ivezic \& Elitzur 1997) to compute the temporal evolution of the dust temperature profile of the contracting core by assuming that gas and dust temperatures are coupled. As shown in Fig. 1, the dust and gas temperatures are decoupled and can differ by up to $4 \mathrm{~K}$. We checked at posteriori that variations in gas phase temperatures of $4 \mathrm{~K}$ only induce small variations in abundances of gaseous species by $30 \%$ at most. The thermal structure of the core is derived from a slab geometry in which the core is irradiated by the interstellar radiation field (ISRF) with a spectrum taken from Evans et al. (2001) and assuming a fixed temperature at the edge of core of $13 \mathrm{~K}$ following the observed temperature profile of L1544. 


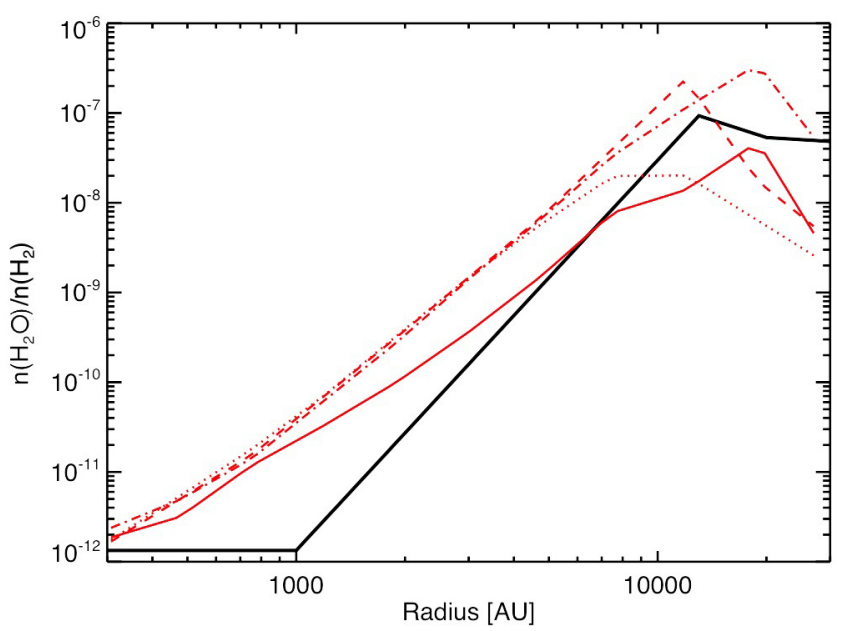

Fig. 4. Water abundance profile in L1544 modelled with GRAINOBLE (red curves) compared with the observed profile based on LIME radiative transfer simulations (black). Dashed-dotted line: $G_{0}=1, A_{\mathrm{V} \text {,ext }}=2$, $t_{\mathrm{c}}=7.5 \times 10^{5} \mathrm{yr}$; dashed line: $G_{0}=1, A_{\mathrm{V}, \mathrm{ext}}=1, t_{\mathrm{c}}=7.5 \times 10^{5} \mathrm{yr}$; dotted line: $G_{0}=0.1, A_{\mathrm{V}, \text { ext }}=1, t_{\mathrm{c}}=7.5 \times 10^{5} \mathrm{yr}$; solid line: $G_{0}=1$, $A_{\mathrm{V}, \mathrm{ext}}=2, t_{\mathrm{c}}=1.5 \times 10^{6} \mathrm{yr}$.

As discussed in Taquet et al. (2013a, 2014), the abundance and the deuteration of the main ice components like water are known to depend on various physical and chemical parameters that are either poorly constrained or that show distributions of values. To reproduce the water abundance profile deduced by the LIME radiative transfer study, we varied the values of three poorly constrained physical parameters that are thought to have a strong impact on the abundance of gaseous water:

1. The external visual extinction $A_{\mathrm{V} \text {,ext }}$ that influences the radius where the water abundance reaches its maximal value. As shown in Fig. 4, decreasing $A_{\mathrm{V} \text {,ext }}$ from two to one magnitude enhances the photodissociation of water at the edge of the core, shifting its maximal abundance toward the core centre from $1.8 \times 10^{4}$ to $1.2 \times 10^{4} \mathrm{AU}$.

2. The external ISRF (interstellar radiation field) $G_{0}$. The photo-desorption rate of water increases with the flux of external UV photons, which is proportional to $G_{0}$. The maximal abundance of water starts to increase with $G_{0}$ at low $G_{0}$ and then decreases when the photo-dissociation of gaseous water overcomes its photo-desorption. Decreasing $G_{0}$ from 1 to 0.1 decreases the maximun water abundance from $2 \times 10^{-7}$ to $2 \times 10^{-8}$.

3. The contraction timescale $t_{\mathrm{c}}$. The core contraction timescale impacts the total number of particles that freeze-out on grains at the centre of the dense core but also the number of particles that are photo-evaporated at lower densities and visual extinctions in the outer shells. The increase in the core contraction timescale between $7.5 \times 10^{5}$ and $1.5 \times 10^{6} \mathrm{yr}$ decreases the water abundance in the dense part of the core, owing to higher depletion, but also slightly increases the water abundance towards the edge because of the higher total number of photo-evaporated water molecules.

The water deuteration profiles obtained for the three sets of parameters used to model the water abundance profile in Fig. 4 are shown in Fig. 5. The water deuteration weakly depends on the physical parameters: the $[\mathrm{HDO}] /\left[\mathrm{H}_{2} \mathrm{O}\right]$ and $\left[\mathrm{D}_{2} \mathrm{O}\right] /\left[\mathrm{H}_{2} \mathrm{O}\right]$ abundance ratios tend to increase towards the centre of the core up to $\sim 25 \%$ and $\sim 8 \%$, respectively. As comprehensively studied

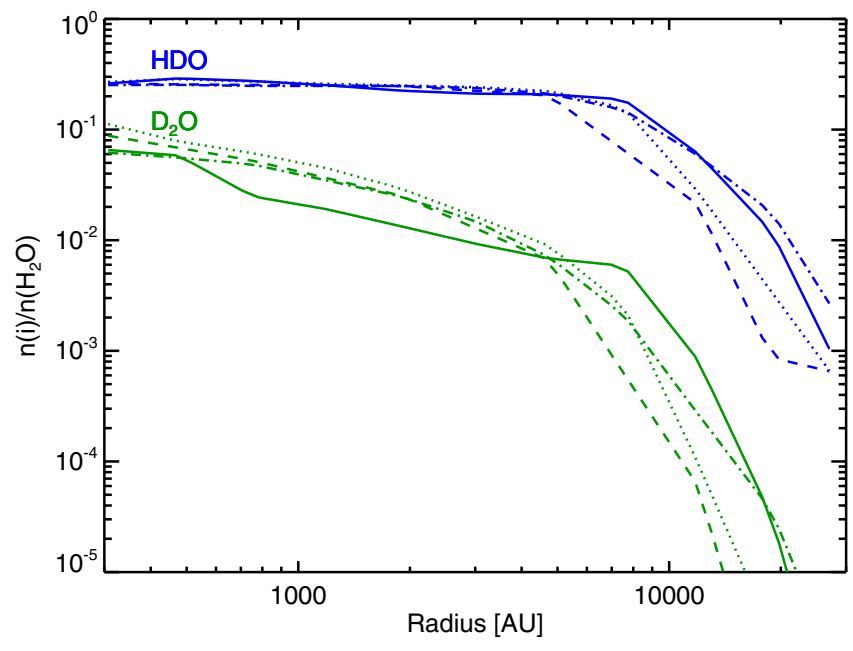

Fig. 5. $[\mathrm{HDO}] /\left[\mathrm{H}_{2} \mathrm{O}\right]$ (blue) and $\left[\mathrm{D}_{2} \mathrm{O}\right] /\left[\mathrm{H}_{2} \mathrm{O}\right]$ (green) deuteration profile in L1544 modelled with GRAINOBLE. Dashed-dotted line: $G_{0}=1$, $A_{\mathrm{V}, \text { ext }}=2, t_{\mathrm{c}}=7.5 \times 10^{5} \mathrm{yr}$; dashed line: $G_{0}=1, A_{\mathrm{V}, \mathrm{ext}}=1$, $t_{\mathrm{c}}=7.5 \times 10^{5} \mathrm{yr}$; dotted line: $G_{0}=0.1, A_{\mathrm{V}, \mathrm{ext}}=1, t_{\mathrm{c}}=7.5 \times 10^{5} \mathrm{yr}$; solid line: $G_{0}=1, A_{\mathrm{V}, \text { ext }}=2, t_{\mathrm{c}}=1.5 \times 10^{6} \mathrm{yr}$.

in previous analyses (Roberts et al. 2004; Flower et al. 2006; Taquet et al. 2014; Sipilä et al. 2015), the increase in the deuteration both in the gas phase and on ices toward the core centre is due to the decrease in both the $\mathrm{CO}$ gas phase abundance and the $\mathrm{H}_{2}$ ortho/para ratio, the two main parameters limiting the deuterium chemistry, with the increase in the total density and the decrease in the temperature. As also discussed in Taquet et al. (2014), the gas phase deuteration of water obtained at the centre of dense cores is higher by more than one order of magnitude than the overall deuteration predicted in interstellar ices. The gas phase $\mathrm{D} / \mathrm{H}$ abundance ratio of water reflects the gas phase chemistry and surface chemistry in the outermost ice layers, in interaction with the gas phase, that are occurring in dense and cold conditions. The low deuteration of water ice is due to its early formation in the molecular cloud phase when the $\mathrm{CO}$ abundance and the $\mathrm{H}_{2}$ ortho/para ratio were high.

Finally, the parameters of the chemical model that best fit the LIME-deduced water abundance profile are $G_{0}=1, A_{\mathrm{V} \text {,ext }}=2$, and $t_{\mathrm{c}}=1.5 \times 10^{6} \mathrm{yr}$ for a fixed diameter of grain $a_{\mathrm{d}}$ assumed to be equal to $0.1 \mu \mathrm{m}$ (see Fig. 4).

\section{HDO modelling with LIME}

Coutens et al. (2012) have shown that in the outer part of the envelope of the low-mass protostar IRAS 16293-2422, the $[\mathrm{HDO}] /\left[\mathrm{H}_{2} \mathrm{O}\right]$ ratio is about $0.2-2.2 \%$ and $\sim 4.8 \%$ in the external layer. This layer can be associated to the parental cloud, a remnant of the initial prestellar core of the protostar. Thus, we can say that an expected realistic $[\mathrm{HDO}] /\left[\mathrm{H}_{2} \mathrm{O}\right]$ ratio in $\mathrm{L} 1544$ may be close to these values. Nonetheless, observed $\mathrm{D} / \mathrm{H}$ ratios for other gaseous species, as well as astrochemical models of starless cores, suggest much higher values, ranging from $7.5 \%$ to $22.5 \%$. We chose to consider six different $\mathrm{D} / \mathrm{H}$ ratios:

- $0.75 \%$ and $2.25 \%$, in agreement with the $[\mathrm{HDO}] /\left[\mathrm{H}_{2} \mathrm{O}\right]$ ratios found by Coutens et al. (2012) in the external layer of IRAS 16293-2422, a low-mass protostar.

- $7.5 \%$ and $22.5 \%$, in agreement with other observed $\mathrm{D} / \mathrm{H}$ ratios in molecules, such as $\mathrm{HCO}^{+}, \mathrm{N}_{2} \mathrm{H}^{+}$, and $\mathrm{H}_{2} \mathrm{CO}$ in 

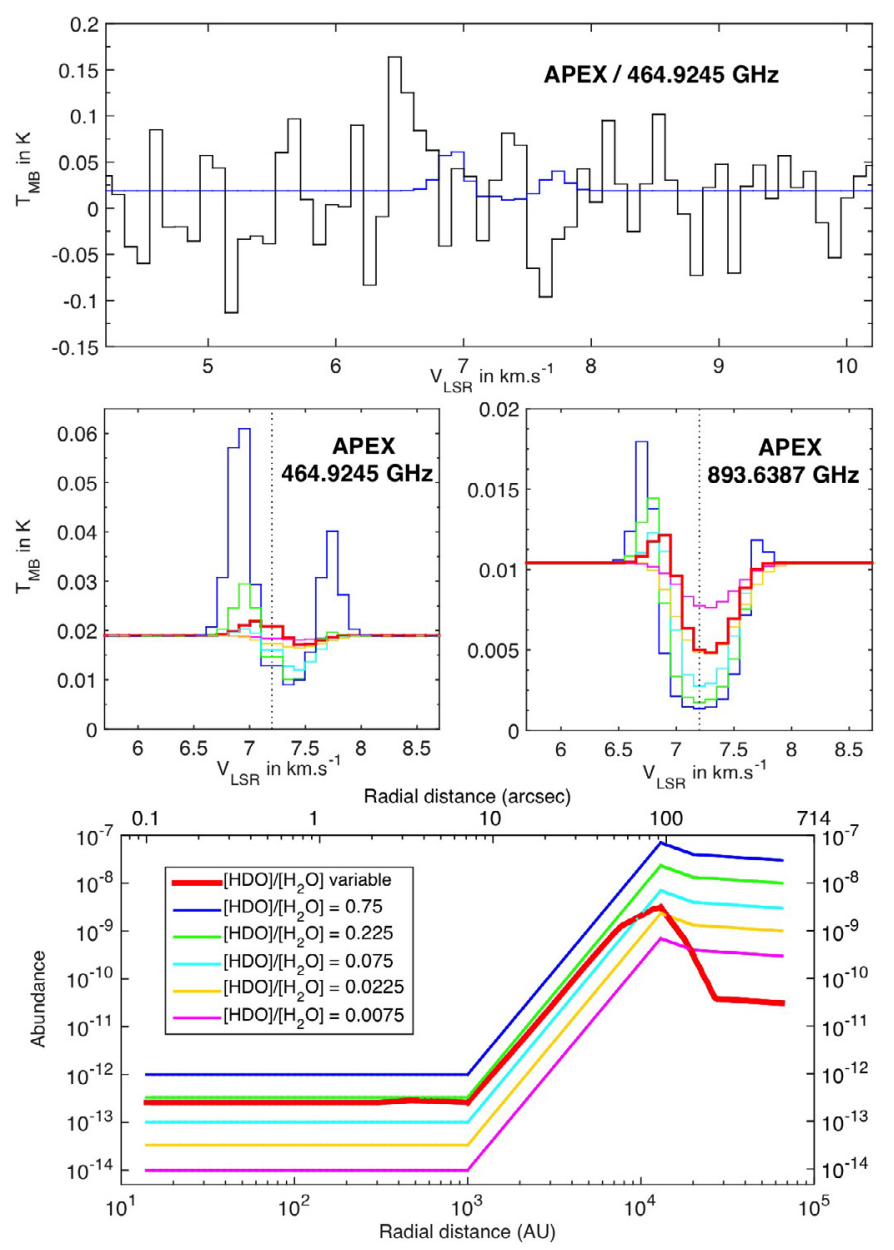

Fig. 6. Top panel: HDO $1_{0,1}-0_{0,0}$ line profile of the $\mathrm{D} / \mathrm{H}=1$ model (in blue) versus APEX observation (in black). Middle panels: HDO $1_{0,1}-$ $0_{0,0}$ (left panel) and $1_{1,1}-0_{0,0}$ (right panel) line profiles for the six different $\mathrm{D} / \mathrm{H}$ ratios. Bottom panel: $\mathrm{HDO}$ abundance profiles as a function of the $\mathrm{D} / \mathrm{H}$ ratio (see text, Sect. 5) used for these models as a function of the radial distance from the core in arcsec (top axis) and AU (bottom axis).

prestellar cores (e.g. Bacmann et al. 2003; Pagani et al. 2007; Kong et al. 2015).

- variable as a function of the radius. We took the $\mathrm{D} / \mathrm{H}$ ratio found by the dotted astro-chemical modelling plotted in Fig. 5. This chemical modelling corresponds to $G_{0}=0.1$, $A_{\mathrm{V}, \mathrm{ext}}=1$, and $t_{\mathrm{c}}=7.5 \times 10^{5} \mathrm{yr}$.

- $75 \%$ to consider an extreme case where the $[\mathrm{HDO}] /\left[\mathrm{H}_{2} \mathrm{O}\right]$ ratio is very high.

Based on our best-fit ad hoc $\mathrm{H}_{2} \mathrm{O}$ abundance profile, we derived the abundance profile for the six previous different $\mathrm{D} / \mathrm{H}$ ratios we consider. We modelled the HDO $1_{0,1}-0_{0,0}$ transition at $464.914 \mathrm{GHz}$ and the $1_{1,1}-0_{0,0}$ transition at $893.639 \mathrm{GHz}$. The APEX observation of the $464.914 \mathrm{GHz}$ transition does not show any detection of HDO towards the source (as seen in the upper panel of Fig. 6), and the $893.639 \mathrm{GHz}$ transition has not been observed. The predicted continuum value at $464.914 \mathrm{GHz}$ and $893.639 \mathrm{GHz}$ are $18.9 \mathrm{mK}$ and $10.4 \mathrm{mK}$, respectively. The predicted continuum value is lower at a higher frequency, which is consistent with a cold prestellar core such as L1544. The two middle panels of Fig. 6 show the spectra of the $1_{0,1}-0_{0,0}$ and $1_{1,1}-0_{0,0}$ transition derived from the output brightness map of the six different models, and the lower panel displays the
Table 1. Intensity of the emission and absorption feature of the two transitions with respect to their continuum level.

\begin{tabular}{cc|c|c|c}
\hline \hline $\begin{array}{c}\mathrm{D} / \mathrm{H} \text { ratio } \\
\text { (in \%) }\end{array}$ & \multicolumn{2}{c|}{$\begin{array}{c}\mathrm{HDO}\left(1_{0,1}-0_{0,0}\right) \\
464.914 \mathrm{GHz}\end{array}$} & \multicolumn{2}{c}{$\begin{array}{c}\mathrm{HDO}\left(1_{1,1}-0_{0,0}\right) \\
893.639 \mathrm{GHz}\end{array}$} \\
\hline & $\begin{array}{c}\text { absorption } \\
\text { (in mK) }\end{array}$ & $\begin{array}{c}\text { emission } \\
\text { (in mK) }\end{array}$ & $\begin{array}{c}\text { absorption } \\
\text { (in mK) }\end{array}$ & $\begin{array}{c}\text { emission } \\
\text { (in mK) }\end{array}$ \\
\hline variable $^{a}$ & 1.9 & 2.9 & 5.4 & 1.5 \\
$75 \%$ & 9.9 & 42.1 & 9.0 & 4.8 \\
$22.5 \%$ & 8.8 & 10.6 & 8.7 & 1.5 \\
$7.5 \%$ & 6.9 & 1.4 & 7.7 & 1.3 \\
$2.25 \%$ & 2.5 & 0.04 & 5.5 & 0.04 \\
$0.75 \%$ & 0.8 & 0.007 & 2.6 & 0.002 \\
\hline
\end{tabular}

Notes. ${ }^{(a)} \mathrm{D} / \mathrm{H}$ ratio as a function of the radius, derived from the dotted astro-chemical modelling shown in Fig. 5. ${ }^{(b)}$ Absorption with respect to the continuum value of $18.9 \mathrm{mK}$ at $464.914 \mathrm{GHz}$ and $10.4 \mathrm{mK}$ at $893.639 \mathrm{GHz}$.

related HDO abundance profile. The upper panel compares the APEX observations of the $464.914 \mathrm{GHz}$ transition and the $\mathrm{D} / \mathrm{H}=1$ model. This comparison clearly shows the limits of detectability for deuterated water with single-dish telescopes such a APEX, even if we consider a very high and unrealistic ratio. Table 1 shows the intensity of the emission and absorption feature for every model with respect to the continuum level of the line.

By looking at the brightness map of any of the previous models, one can note that the absorption feature of the HDO lines only shows up on a 50" scale around the centre of the model. If we consider the most compact Cycle 3 configuration of ALMA (C36-1), it is impossible to entirely map L1544 in a convenient number of pointings owing to the size of the core ( 20 $000 \mathrm{AU}$, which means $\sim 143^{\prime \prime}$ at $140 \mathrm{pc}$ ), and even if we consider only the absorption feature $\left(\sim 50^{\prime \prime}\right)$, it would still require too many pointings since the antenna beamsize of the C36-1 configuration is $\sim 12.5^{\prime \prime}$ and $\sim 6.5^{\prime \prime}$ for the two HDO transitions. The ALMA interferometer is not optimum here to detect a very extended emission, and complementary ACA observations will not be sufficient to cover the missing $(u, v)$-plane observations. Only a single-dish telescope can give a convenient beam size to cover at least the absorption feature of L1544, but right now, such low sensitivities ( $\sim 2 \mathrm{mK}$ at $464.914 \mathrm{GHz}$ and $\sim 5 \mathrm{mK}$ at $893.639 \mathrm{GHz}$ ) cannot be reached with ground-based observatories, and no space observatory is foreseen in the future.

\section{Conclusions}

Based on the recent detection of the $1_{1,0}-1_{0,1}$ water transition using the Herschel/HIFI instrument, we used the APEX observatory to constrain the water fractionation in the L1544 prestellar core. We used LIME to model the $1_{1,0}-1_{0,1} \mathrm{H}_{2} \mathrm{O}$ and $1_{0,1}-0_{0,0}$ HDO line profiles towards L1544 with a full radiative transfer treatment in 3D. Keto et al. (2014) derived the density, temperature, and velocity profile of the source. However, using LIME instead of MOLLIE, we found that their abundance profile predicts an emission that is 3.5 times stronger than the observation while using their deduced dust opacity, LIME predicts a continuum emission at $557 \mathrm{GHz}$ about two times stronger than the observed value.

This result points to the need for a detailed comparison between these codes for the specific case of water in prestellar 
cores. We have found an ad hoc abundance profile that fits the Herschel/HIFI $\mathrm{H}_{2} \mathrm{O}$ observation better using LIME. We also found a new estimation of the dust opacity to reproduce the observed continuum of $\sim 10.2 \mathrm{mK}$. We used a detailed chemical modelling using both gas-phase and grain-surface chemistry to reproduce the water profile and predict its subsequent deuterated water profile. The resulting profile $\left(G_{0}=1, A_{\mathrm{V} \text {,ext }}=2\right.$, $\left.t_{\mathrm{c}}=1.5 \times 10^{6} \mathrm{yr}, a_{\mathrm{d}}=0.1 \mu \mathrm{m}\right)$ has then been compared with our APEX observations. Our study shows the limit of detectability for deuterated water in prestellar cores using ground-based facilities with both single-dish telescopes and interferometric antennas.

Acknowledgements. The authors are grateful to Carlos De Breuck for his help during the APEX observations. C.C. acknowledges the financial support by the French Space Agency CNES. P.C. acknowledges the financial support of the European Research Council (ERC; project PALs 320620).

\section{References}

Aikawa, Y., Wakelam, V., Hersant, F., Garrod, R. T., \& Herbst, E. 2012, ApJ, 760,40

Bacmann, A., Lefloch, B., Ceccarelli, C., et al. 2003, ApJ, 585, L55

Bizzocchi, L., Caselli, P., Spezzano, S., \& Leonardo, E. 2014, A\&A, 569, A27

Bockelée-Morvan, D., Biver, N., Crovisier, J., et al. 2014, A\&A, 562, A5

Boogert, A., Gerakines, P., \& Whittet, D. 2015, ARA\&A, 53, 541

Brinch, C., \& Hogerheijde, M. R. 2010, A\&A, 523, A25

Brünken, S., Sipilä, O., Chambers, E. T., et al. 2014, Nature, 516, 219

Carr, J. S., \& Najita, J. R. 2008, Science, 319, 1504

Caselli, P., \& Ceccarelli, C. 2012, A\&ARv, 20, 56

Caselli, P., Walmsley, C. M., Zucconi, A., et al. 2002, ApJ, 565, 331

Caselli, P., van der Tak, F. F. S., Ceccarelli, C., \& Bacmann, A. 2003, A\&A, 403, L37

Caselli, P., Keto, E., Pagani, L. et al. 2010, A\&A, 521, L29

Caselli, P., Keto, E., Bergin, E. A., et al. 2012, ApJ, 759, L37

Ceccarelli, C., Caselli, P., Bockelée-Morvan, D., et al. 2014, Protostars and Planets VI, 859

Coutens, A., Vastel, C., Caux, E., et al. 2012, A\&A, 539, A132

Coutens, A., Vastel, C., Cabrit, S., et al. 2013, A\&A, 560, A39
Coutens, A., Vastel, C., Hincelin, U., et al. 2014, MNRAS, 445, 1299

Crapsi, A., Caselli, P., Walmsley, C. M., et al. 2005, ApJ, 619, 379

Crapsi, A., Caselli, P., Walmsley, M. C., \& Tafalla, M. 2007, A\&A, 470, 221

Dartois, E., Thi, W.-F., Geballe, T. R., et al. 2003, A\&A, 399, 1009

van Dishoeck, E. F., Bergin, E. A., Lis, D. C., \& Lunine, J. I. 2014, Protostars and Planets VI, 835

Dubernet, M.-L., Daniel, F., Grosjean, A., \& Lin, C. Y. 2009, A\&A, 497, 911

Evans, N. J., II, Rawlings, J. M. C., Shirley, Y. L., \& Mundy, L. G. 2001, ApJ, 557,193

Flower, D. R., Pineau Des Forêts, G., \& Walmsley, C. M. 2006, A\&A, 449, 621

Hasegawa, T. I., \& Herbst, E. 1993a, MNRAS, 261, 83

Hasegawa, T. I., \& Herbst, E. 1993b, MNRAS, 263, 589

Hasegawa, T. I., Herbst, E., \& Leung, C. M. 1992, ApJS, 82, 167

Hogerheijde, M., \& van der Tak, F. 2000, Astrophysics Source Code Library, 0008.002

Hollenbach, D., Kaufman, M. J., Bergin, E. A., \& Melnick, G. J. 2009, ApJ, 690, 1497

Ivezic, Z., \& Elitzur, M. 1997, MNRAS, 287, 799

Keto, E. R. 1990, ApJ, 355, 190

Keto, E., \& Caselli, P. 2010, MNRAS, 402, 1625

Keto, E., \& Rybicki, G. 2010, ApJ, 716, 1315

Keto E., Rawlings J., Caselli P. 2014, MNRAS, 440, 2616, 301

Kong, S., Caselli, P., Tan, J. C., Wakelam, V., \& Sipilä, O. 2015, ApJ, 804, 98

Ossenkopf, V., \& Henning, T. 1994, A\&A, 291, 943

Pagani, L., Bacmann, A., Cabrit, S., \& Vastel, C. 2007, A\&A, 467, 179

Parise, B., Simon, T., Caux, E., et al. 2003, A\&A, 410, 897

Persson, M. V., Jørgensen, J. K., van Dishoeck, E. F., \& Harsono, D. 2014, A\&A, 563, A74

Podio, L., Kamp, I., Codella, C., et al. 2013, ApJ, 766, L5

Roberts, H., Herbst, E., \& Millar, T. J. 2004, A\&A, 424, 905

Sipilä, O., Caselli, P., \& Harju, J. 2013, A\&A, 554, A92

Sipilä, O., Caselli, P., \& Harju, J. 2015, A\&A, 578, A55

Taquet, V., Ceccarelli, C., \& Kahane, C. 2012, A\&A, 538, A42

Taquet, V., Peters, P. S., Kahane, C., et al. 2013a, A\&A, 550, A127

Taquet, V., López-Sepulcre, A., Ceccarelli, C., et al. 2013b, ApJ, 768, L29

Taquet, V., Charnley, S. B., \& Sipilä, O. 2014, ApJ, 791, 1

Troscompt, N., Faure, A., Maret, S., et al. 2009, A\&A, 506, 1243

Vastel, C., Caselli, P., Ceccarelli, C., et al. 2006, ApJ, 645, 1198

Vastel, C., Ceccarelli, C., Lefloch, B., \& Bachiller, R. 2014, ApJ, 795, LL2

Vasyunin, A. I., \& Herbst, E. 2013, ApJ, 769, 34

Wakelam, V., Vastel, C., Aikawa, Y., et al. 2014, MNRAS, 445, 2854

Zucconi, A., Walmsley, C. M., \& Galli, D. 2001, A\&A, 376, 650 